\title{
Disruption of KCNQ1 prevents methylation of the ICR2 and supports the hypothesis that its transcription is necessary for imprint establishment
}

\author{
Jasmin Beygo $^{1} \cdot$ Joachim Bürger $^{2} \cdot$ Tim M. Strom $^{3} \cdot$ Sabine Kaya ${ }^{1} \cdot$ Karin Buiting $^{1}$
}

Received: 4 September 2018 / Revised: 7 January 2019 / Accepted: 2 February 2019 / Published online: 18 February 2019

(c) European Society of Human Genetics 2019

\begin{abstract}
Beckwith-Wiedemann syndrome (BWS; OMIM \#130650) is an imprinting disorder caused by genetic or epigenetic alterations of one or both imprinting control regions on chromosome 11p15.5. Hypomethylation of the centromeric imprinting control region (KCNQ1OT1:TSS-DMR, ICR2) is the most common molecular cause of BWS and is present in about half of the cases. Based on a BWS family with a maternal deletion of the 5' part of $K C N Q 1$ we have recently hypothesised that transcription of $K C N Q 1$ is a prerequisite for the establishment of methylation at the KCNQ1OTI:TSSDMR in the oocyte. Further evidence for this hypothesis came from a mouse model where methylation failed to be established when a poly(A) truncation cassette was inserted into this locus to prevent transcription through the DMR. Here we report on a family where a balanced translocation disrupts the $K C N Q 1$ gene in intron 9. Maternal inheritance of this translocation is associated with hypomethylation of the KCNQ1OT1:TSS-DMR and BWS. This finding strongly supports our previous hypothesis that transcription of $K C N Q 1$ is required for establishing the maternal methylation imprint at the KCNQ1OT1:TSS-DMR.
\end{abstract}

\section{Introduction}

Beckwith-Wiedemann syndrome (BWS; OMIM \#130650) is an overgrowth disorder with a highly variable clinical presentation including macrosomia, macroglossia, organomegaly, abdominal wall defects, hemihypertrophy, earlobe creases and an elevated risk for childhood tumours. The disease is caused by different genetic and epigenetic alterations affecting one or both of the two imprinted domains on chromosome 11p15.5 [1, 2].

Supplementary information The online version of this article (https:// doi.org/10.1038/s41431-019-0365-x) contains supplementary material, which is available to authorized users.

Jasmin Beygo

jasmin.beygo@uni-due.de

1 Institut für Humangenetik, Universitätsklinikum Essen, Universität Duisburg-Essen, Essen, Germany

2 Limbach Humangenetik Berlin Kudamm, Berlin, Germany

3 Institute of Human Genetics, Helmholtz Zentrum München, Neuherberg, Germany
These two imprinted domains are each regulated separately by its own imprinting control region (ICR1 and ICR2): the telomeric $H 19 / I G F 2$ :IG-differentially methylated region (DMR) and the centromeric KCNQ1OT1:TSSDMR, respectively.

The KCNQ1OT1:TSS-DMR is located in intron 10 of the $K C N Q 1$ gene and serves as a promoter for the long non-coding antisense transcript KCNQ1OT1 (Fig. 1). The DMR is methylated on the maternal allele and regulates the imprinted expression of several maternally expressed genes (e.g. $K C N Q 1$ and $C D K N 1 C$ ) and the paternally expressed KCNQ1OT1 in the imprinted domain [3].

The most frequent molecular defect (50-60\%) in patients with BWS is loss of methylation (hypomethylation) at the KCNQ1OT1:TSS-DMR. Less frequent causes are paternal uniparental disomy of 11p15.5 ( 20-25\%), gain of methylation (hypermethylation) at the H19/IGF2:IG-DMR ( 5-10\%), paternal duplications $(\sim 1-2 \%)$ or disease causing variants in CDKN1C $(\sim 5 \%)$ [3, 4]. In rare cases, maternal deletions affecting the H19/IGF2:IG-DMR and the KCNQ1OT1:TSSDMR, as well as chromosomal rearrangements, have been identified as causative for BWS [5-8].

Interestingly, one $\sim 50 \mathrm{~kb}$ familial duplication, which involves the $3^{\prime}$ part of intron 1 , exon 2 and the $5^{\prime}$ part of 


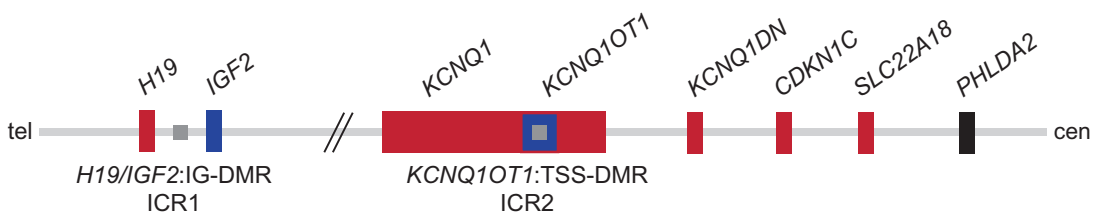

Fig. 1 Overview of the imprinted domains on chromosome 11p15.5. Given are the two imprinting control regions (ICRs) on chromosome 11p15.5. The centromeric KCNQ1OT1:TSS-DMR is methylated on the maternal allele (grey square). It regulates the expression of the maternally expressed genes $K C N Q 1, K C N Q 1 D N, C D K N 1 C$ and

intron 2 of the KCNQ1 gene but not the KCNQ1OT1:TSSDMR itself, leads to absence of methylation of the DMR and BWS when maternally inherited [9]. Furthermore, we recently reported a family with multiple affected offspring where a maternally inherited deletion spanning the promoter and the 5' part of $K C N Q 1$ but not the KCNQ1OT1:TSS-DMR leads to absence of methylation at the DMR and BWS [10]. The deletion described by us as well as the duplication described by Demars et al. probably interfere with $K C N Q 1$ expression in the oocyte. Based on these findings we hypothesised that transcription of KCNQ1 through the KCNQ1OT1:TSS-DMR is required for the establishment of the methylation imprint in the maternal germline [10].

We here report on a familial case of BWS with absence of methylation at the KCNQ1OT1:TSS-DMR due to a complex chromosomal structural rearrangement involving chromosome 11 and 13. A translocation disrupts $K C N Q 1$ and relocates exons $1-9$ of the gene to the derivative chromosome 13 while exons 10-15 including the KCNQ1OT1:TSSDMR remain on chromosome 11. Thus, transcription of $K C N Q 1$ does not pass through the DMR. As a consequence methylation at this DMR is not established in the mothers' germline leading to BWS in the child.

\section{Material and methods}

\section{Samples}

A native chorionic villi sample and peripheral blood samples from the mother, father and maternal grandparents were obtained after written informed consent. The study was approved by the ethics committee of the University Duisburg-Essen. Information regarding this family has been submitted to the LOVD (patient ID 00180299; http://databa ses.lovd.nl/shared/individuals).

\section{DNA extraction}

DNA was extracted using the blood and cell culture and the Flexigene Kit (Qiagen, Hilden, Germany) following the manufacturer's protocol for the different tissue types.
SLC22A18 (depicted in red) and the paternally expressed KCNQ1OT1 (blue). The biallelically expressed gene PHLDA2 is shown in black. The telomeric H19/IGF2:IG-DMR is methylated on the paternal allele with the maternally expressed $H 19$ and the paternally expressed $I G F 2$ gene. Tel telomeric, cen centromeric. Modified from Beygo et al. 2016

Maternal contamination was excluded in all fetal samples using the Powerplex 16 Kit (Promega, Mannheim, Germany) prior to the subsequently described analyses.

\section{Methylation-specific multiplex ligation-dependent probe amplification (MS-MLPA)}

Gene dosage and methylation analyses of the chromosomal region $11 \mathrm{p} 15$ including the two ICRs were carried out using the SALSA MS-MLPA Kit ME030-C3. To exclude multiple methylation defects we also conducted MS-MLPA for several imprinted loci using the ME034-A1 kit (MRC Holland, Amsterdam, Netherlands). Both MS-MLPAs were performed according to the manufacturer's manual. Amplification products were analysed on an ABI3130XL capillary sequencer (Applied Biosystems, Darmstadt, Germany) followed by data analysis with the Gene Marker Software (Softgenetics, State College, PA, USA).

\section{SNP array analysis}

To identify genome-wide copy number variants/aberrations, SNP array analysis was conducted using the CytoScan HD Array (Affymetrix, Santa Clara, CA, USA) following the manufacturer's protocol. Data analysis was conducted with the Chromosome Analysis Suite software (ChAS, v3.1.0.15, Affymetrix).

\section{Whole genome sequencing (WGS)}

WGS was conducted in the mother (II-2) as described previously [10]. Briefly, the library was prepared using PCR-free protocols (TruSeq DNA PCR-Free, Illumina, San Diego, CA, USA) and sequencing was performed on the HiSeq2000/2500 systems (Illumina).

\section{Breakpoint verification}

Breakpoints were verified by junction PCR. Additionally, the presence of the normal chromosomes was verified by PCRs including the breakpoint regions. All PCRs were conducted using standard protocols. Primer sequences are 
available upon request. Amplified products were sequenced using the ABI3130XL capillary sequencer (Applied Biosystems, Darmstadt, Germany). For sequence analysis the Geneious software (Biomatters, Auckland, New Zealand) was utilised.

\section{Results}

A 34-year-old woman was referred to genetic counselling because of four unsuccessful pregnancies, which ended with one spontaneous and three missed abortions between the eighth and eleventh week of gestation. The couple subsequently underwent two procedures of IVF, but after embryo transfer the implantation failed. It was reported that only 2 and 1 oocytes, respectively, were of sufficient quality for IVF. The woman and her partner are healthy, nonconsanguineous and have no living children. The family history was unremarkable. Cytogenetic analysis on chorionic villi at week 11 from a missed abortion obtained by curettage revealed the presence of a complex structural rearrangement with a translocation and an inversion affecting chromosome 11 and 13 ((46,XX,der(11)inv(11) $(\mathrm{p} 15 \mathrm{q} 22) \mathrm{t}(11 ; 13)(\mathrm{p} 15 ; \mathrm{q} 22), \operatorname{der}(13) \mathrm{t}(11 ; 13)(\mathrm{p} 15 ; \mathrm{q} 22))$. The same rearrangement was subsequently found in lymphocytes from the mother. The structural rearrangement in the mother was de novo since her parents have normal karyotypes. No material from the other three pregnancies was available for cytogenetic or molecular genetic analysis.

As one translocation breakpoint appeared to be at chromosome 11p15, which contains the BWS critical region, MS-MLPA for this chromosomal region was performed on the DNA of the mother and the foetus. In the foetus we obtained a normal methylation pattern for the H19/IGF2: IG-DMR but complete absence of methylation of the KCNQ1OT1:TSS-DMR at all four methylation sensitive probes, which is compatible with the molecular diagnosis of BWS (Supplementary Table 1). Gene dosage analysis revealed no evidence for a deletion.

Both the H19-IGF2:IG-DMR (ICR1) and the KCNQ1OT1:TSS-DMR (ICR2) are germline DMRs where the methylation is already set in the paternal and maternal germline, respectively. Methylation studies using cultured and native chorionic villi samples (CVS) showed that the methylation imprint is stable [11]. Only one false-positive result was reported so far in the context of mosaic imprinting defects [12]. However, the native CVS DNA from the foetus we investigated showed a complete absence of methylation and no sign of a methylation mosaic.

The mother had a normal methylation pattern for both imprinting control centres, the H19/IGF2:IG-DMR and the KCNQ1OT1:TSS-DMR, and showed normal gene dosage for all investigated MLPA probes. The same findings were made in the maternal grandparents. SNP array analysis of the mother's DNA did not detect any genetic imbalance.

The complete loss of methylation at the KCNQ1OT1: TSS-DMR in the foetus is caused in cis and thus results in a single locus imprinting defect. However, we have performed additional methylation analyses at several imprinted loci by MS-MLPA to exclude the extremely low probability of a multilocus imprinting defect. As expected, all loci investigated except the KCNQ1OT1:TSS-DMR showed normal methylation values (data not shown).

Whole-genome sequencing allowed us to determine the breakpoints of the complex structural rearrangement on the DNA sequence level. By this we found that one breakpoint (termed BP1) of the balanced translocation between chromosome 11 and 13 maps to position chr13:70,765,320 (hg19) on the long arm of chromosome 13 and to position chr11:2,613,439 on the short arm of chromosome 11 (for details see Fig. 2a). The telomeric region of $13 \mathrm{q}$ (chr13:70,765,321_qter) was fused to chromosome 11. Due to a large inversion, which affects the majority of the translocation chromosome 11 (inverted region: chr11:2,613,440_99, 998,486), the chromosome 13 material was fused to the telomeric region of the long arm of chromosome 11 (BP2), whereas a third breakpoint (BP3) represents the fusion at the short arm of chromosome 11 with the terminal end of the long arm of this chromosome at positions chr11:2,613,440 and chr11:99,998,487, respectively (hg19, [chr13:pter_cen_ 70765321]::[chr11:2613439inv_pter] and [chr11:pter_2613440_ 99998486inv]::[chr13:70765320_qter]). As a consequence of the translocation, the $K C N Q 1$ gene and its antisense gene KCNQ1OT1 are disrupted. The breakpoint maps inside intron 9 of $K C N Q 1$ and the 5' region (exons 1-9) is translocated to the derivative chromosome 13 at the BP1 region, whereas the 3 ' region including the differentially methylated region of KCNQ1OT1 stays on chromosome 11 at the BP3 region (Fig. 2b). All breakpoints were verified by junction PCRs in the mother and the foetus.

The normal methylation pattern in the MS-MLPA detected in the mother suggests that the disrupted $K C N Q 1$ copy is located on the paternal allele. To prove this we searched for informative SNPs in the BP regions on chromosome 11 and 13 in the mother and her parents and found a rare informative variant near the $\mathrm{BP} 2$ region (Fig. 3). The segregation of the variant clearly showed that the $K C N Q 1$ copy disrupted by the rearrangement in the mother was of paternal origin.

\section{Discussion}

A role for transcription in the establishment of DNA methylation at differentially methylated regions (DMRs) in the mouse oocyte was demonstrated previously for different 


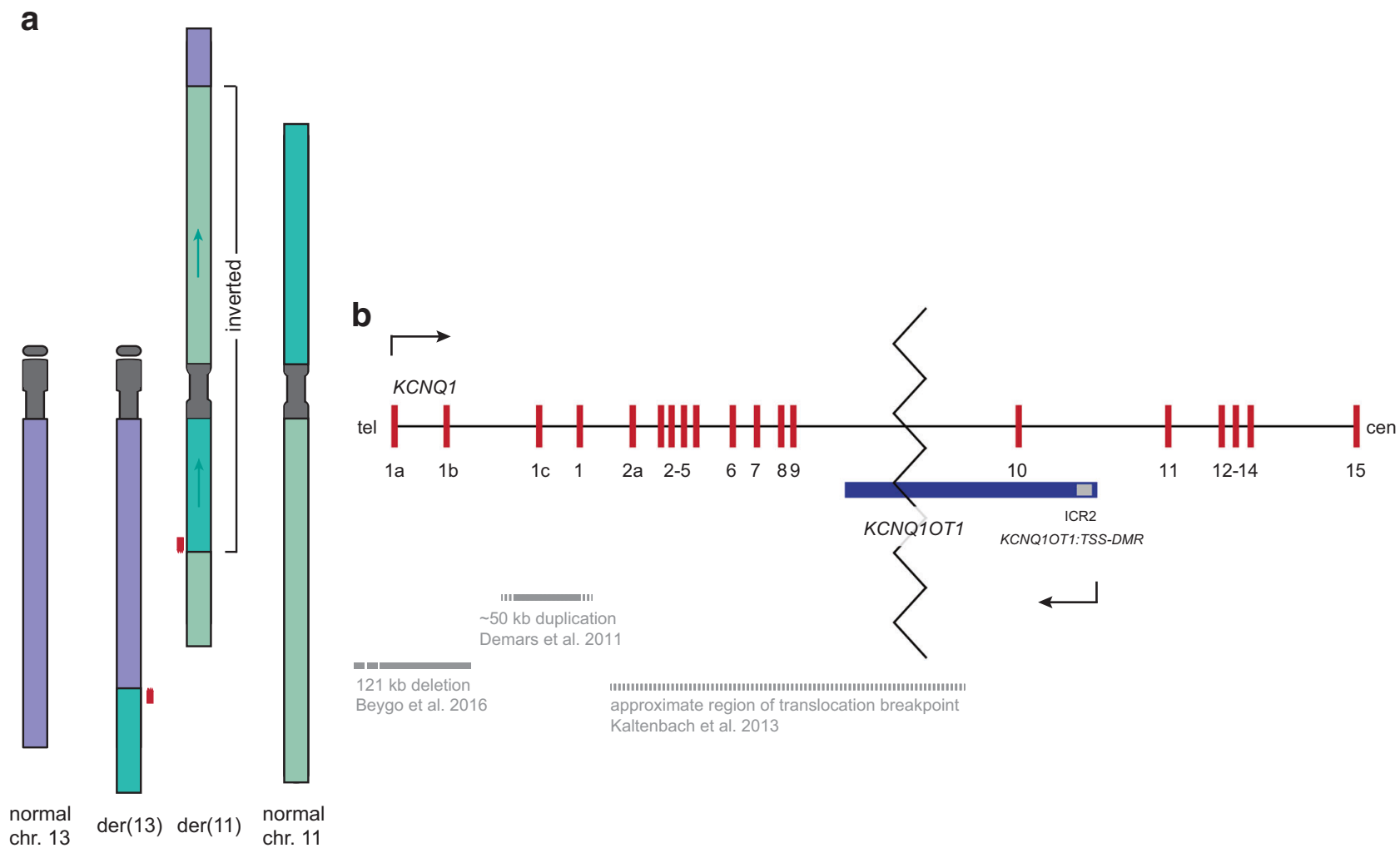

Fig. 2 a Schematic view of the chromosomal rearrangement and b detail view of the disruption of KCNQ1 and KCNQ1OT1. In part a the chromosomes 13 (purple) and 11 (green, 11p dark green, $11 \mathrm{q}$ lighter green) are given in the normal state (outer chromosomes) and with the rearrangement detected in the family (inner chromosomes). The terminal part of $13 \mathrm{q}$ is translocated to $11 \mathrm{q}$ while the terminal part of $11 p$ is translocated to $13 q$. Furthermore, the biggest part of the derivative chromosome 11 is inverted (indicated by arrows). No large deletions or duplications associated with the breakpoints have been detected so that the rearrangement is balanced. The breakpoints are localised in 13q21.33, 11p15.5 and 11q22.1 and were confirmed by junction PCRs. The disrupted gene $K C N Q 1$ is indicated as red boxes. Not drawn to scale. Part $\mathbf{b}$ shows the detailed view of the maternally expressed $K C N Q 1$ (red) and its antisense transcript, the paternally

imprinted loci. Kelsey and colleagues could demonstrate this for the first time in 2009 for the Gnas locus [13]. In this study it was shown that disruption of transcription initiated from the further upstream Nesp promoter prevented the establishment of methylation at the maternal germline DMR. Later, the same group obtained similar results for another imprinted locus, Plagll [10]. They could show that loss of Plagll transcription in the mouse oocyte leads to a failure in the establishment of DNA methylation, which results in a completely unmethylated DMR.

Further evidence that transcription is also required for the establishment of maternal imprinting at the murine and human Snrpn/SNRPN loci came from studies on a transgenic mouse model and the finding that the Angelman syndrome imprinting centre, which maps upstream of $S N R P N$, encodes a transcriptional promoter in human expressed $K C N Q 1 O T 1$ (blue). The imprinting control region 2 (ICR2, $K C N Q 1 O T 1: T S S-D M R)$ is given as a grey square. Direction of the transcription is indicated by arrows. From the literature the deletion described in Beygo et al. 2016, the duplication described in Demars et al. 2011 (grey bars) and the region containing the translocation breakpoint described in Kaltenbach et al. 2013 are depicted (gashed grey bar). Tel telomeric, cen centromeric. Modified from Beygo et al. 2016. The translocation of $11 p$ material to chromosome 13q disrupts both transcripts (indicated by zigzag line) and is located in intron nine of KCNQ1. The 5' part of the gene is translocated to chromosome 13 while the 3' part including the ICR2 stays on chromosome 11p. The disruption prevents transcription of $K C N Q 1$ through the ICR2. Not drawn to scale. Tel telomeric, cen centromeric. Figure modified from Beygo et al. 2016

oocytes. Transcription starting from this promoter transits the SNRPN:TSS-DMR [14, 15].

Based on a familial deletion including the promoter and the 5' part of the $K C N Q 1$ gene, which leads to absence of methylation at the KCNQ1OT1:TSS-DMR and to BWS when maternally inherited, we have recently suggested that transcription of $K C N Q 1$ is involved in establishing the maternal methylation imprint of the KCNQ1OT1:TSS-DMR in the oocyte [10]. This hypothesis is further supported by the finding that also a duplication, which affects the 5' part of KCNQ1 but does not include the KCNQ1OT1:TSSDMR, leads to absence of methylation at the DMR and to BWS after maternal transmission [9]. It is possible that both the deletion and the duplication interfere with $K C N Q 1$ expression in the oocyte, maybe by affecting an oocytespecific start site of this gene. 


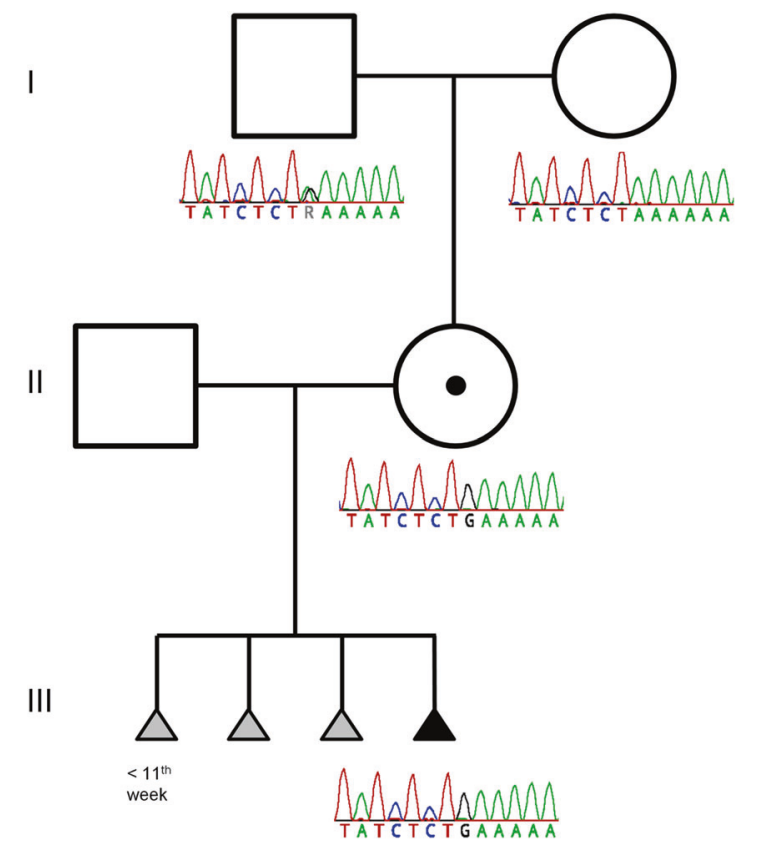

Fig. 3 Pedigree of the family including a variant showing the grandparental inheritance of the derivative chromosome 11. The figure depicts the family pedigree with the results of Sanger-sequencing of the breakpoint region 2 showing the grandparental inheritance of the derivative chromosome 11 . The grandfather and the grandmother have two normal chromosomes. The grandfather is heterozygous for a private variation $(A>G)$, the grandmother is homozygous $A$. For the mother and the fetus (III-4) only the derivative chromosome 11 was amplified using primers on each side of the breakpoint (BP2). The derivative chr.11 in the mother shows the G-allele indicating that the rearrangement involves the paternal allele. This is in accordance with her normal phenotype. She passed the derivative chr.11 on to her child (III-4) indicated again by the G-allele

Moreover, by analysing a mouse model carrying a poly (A) truncation cassette, which blocked Kcnql primary transcripts from reaching the Kcnq1ot1:TSS-DMR, it could be demonstrated that this cassette precludes the establishment of methylation at this DMR when maternally inherited. As a consequence, Kcnq1otl is biallelically expressed and the maternal-only expressed gene $C d k n l c$ is silenced [16].

In the current study we present a family where a complex chromosomal structural rearrangement relocates exons 1-9 of $K C N Q 1$ to the derivative chromosome 13 while exons 10-15 including the KCNQ1OT1:TSS-DMR still remain on chromosome 11. Thus, transcription of KCNQ1 on the maternal allele could not reach the KCNQ1OTI:TSS-DMR, which probably results in a completely unmethylated maternal allele and BWS.

Finally, the previously reported two families and the new family from the study at hand, as well as the mouse model with the poly (A) truncation cassette, provide a direct link between $K C N Q 1$ transcription and the establishment of the methylation mark at the KCNQ1OT1:TSS-DMR $[9,10,16]$. This is consistent with the finding that in a transgenic mouse with a $260 \mathrm{~kb}$ transgene, which contains the Kcnq1otl:TSSDMR and about half of the Kcnql gene but not its promoter, the methylation imprint was not proper set in the female germline [17].

Approximately $50 \%$ of patients with BWS have an epimutation at the KCNQ1OTI:TSS-DMR. Most of them are somatic mosaics for an imprinting defect. However, a subgroup of these patients shows a complete loss of methylation at the KCNQ1OT1:TSS-DMR. It might be possible that this is due to a yet unidentified/unknown deletion or other rearrangement, which disrupts $K C N Q 1$ transcription before it reaches the KCNQ1OT1:TSS-DMR.

$K C N Q 1$ is maternally expressed in most tissues but biallelical expressed in the heart. Heterozygous pathogenic variants but also balanced translocations and deletions affecting $K C N Q 1$ lead to long QT syndrome-1 (LQTS1). LQTS1 is a cardiac arrhythmia syndrome characterised by a prolongation of the QT interval and ventricular arrhythmias. These cardiac arrhythmias can result in syncope, cardiac arrest or sudden death. Two cases of LQTS1 plus BWS have been reported so far. In 2013, Gurrieri et al. described a patient with LQTS1 and mild BWS due to a deletion affecting exons 11-16 of the $K C N Q 1$ gene including the KCNQ1OT1:TSS-DMR [7]. In this case the deletion at the KCNQ1OT1:TSS-DMR itself led to the BWS phenotype. However, Kaltenbach et al. (2013) reported a familial balanced translocation $t$ $(11 ; 17)(\mathrm{p} 15.5 ; \mathrm{q} 21.3)$, which also led to both LQTS1 and BWS when inherited from the mother but led solely to LQTS1 when inherited from the father [18]. Based on fluorescent in situ hybridization this translocation interrupts the $K C N Q 1$ gene between exons 2 and 10 and was shown to cause absence of methylation of the KCNQ1OT1:TSS-DMR on the maternally inherited derivative chromosome 11. This familial case resembles our two families, the one with the deletion of the 5' part of $K C N Q 1$ and the one from this current study with the translocation $\mathrm{t}(11 ; 13)$. Thus, the mothers from these two families are likely affected by LQTS1. That this has not come to attention yet is not surprising as many people with long QT syndrome have no signs or symptoms. Therefore, we recommended that the two mothers undergo a cardiac examination. The mother of the family we described in 2016 has a QTc interval of $460 \mathrm{~ms}$, which is borderline and she was recommended further check-ups (Ivana Joksic, personal communication). By contrast, in the mother from our current family the QTc time at rest ECG is in the normal range. Since neither syncope nor anything else is documented, no long QT syndrome can be clinically diagnosed as it is the case in $36 \%$ of patients [19]. As a consequence from these findings individuals with BWS due to a disruption of $K C N Q 1$ transcription and absence of methylation at the KCNQ1OT1:TSS-DMR 
should be investigated for a pre-existing condition of LQTS1 because of the potential clinical consequences.

Of note, the present family has four unsuccessful consecutive pregnancies and no living offspring. Since all pregnancies resulted in early abortions it is not possible to figure out the cause for the losses. No information regarding a possible phenotype could be obtained as no fetal material from the first three pregnancies was available for genetic analysis. Hence, we cannot exclude that all foetuses have inherited the derivative chromosome 11 from the mother and therefore no functional maternal copy of $K C N Q 1$, which would hint to a segregation distortion. Interestingly, a similar situation was present in the family described in our previous study [10]. In contrast to the current family, the pregnancies lasted longer and a BWS phenotypic feature (giant omphalocele) could be observed in almost all offspring. Additionally, transmission ratios in both families also tend to be skewed in favour of the allele carrying the deletion. We have already discussed in detail a possible segregation distortion and associated mechanisms before [10].

In summary, with the present study we provide further evidence strongly supporting our previous hypothesis that transcription of $K C N Q 1$ trough the DMR is necessary for the establishment of the methylation imprint at the KCNQ1OT1:TSS-DMR.

Acknowledgements We thank the family for their participation in this study and Bernhard Horsthemke for helpful discussions and critical reading of the manuscript. This work was funded by the Bundesministerium für Bildung und Forschung (BMBF; Imprinting diseases, grant No. 01GM1513A).

\section{Compliance with ethical standards}

Conflict of interest The authors declare that they have no conflict of interest.

Publisher's note: Springer Nature remains neutral with regard to jurisdictional claims in published maps and institutional affiliations.

\section{References}

1. Weksberg R, Shuman C, Beckwith JB. Beckwith-Wiedemann syndrome. Eur J Hum Genet. 2010;18:8-14.

2. Brioude F, Kalish JM, Mussa A, Foster AC, Bliek J, Ferrero GB, et al. Expert consensus document: clinical and molecular diagnosis, screening and management of Beckwith-Wiedemann syndrome: an international consensus statement. Nat Rev Endocrinol. 2018;14:229-49.

3. Choufani S, Shuman C, Weksberg R. Molecular findings in Beckwith-Wiedemann syndrome. Am J Med Genet C Semin Med Genet. 2013;163C:131-40.

4. Eggermann T, Algar E, Lapunzina P, Mackay D, Maher ER, Mannens M, et al. Clinical utility gene card for: BeckwithWiedemann Syndrome. Eur J Hum Genet. 2014;22.
5. Begemann M, Spengler S, Gogiel M, Grasshoff U, Bonin M, Betz RC, et al. Clinical significance of copy number variations in the $11 \mathrm{p} 15.5$ imprinting control regions: new cases and review of the literature. J Med Genet. 2012;49:547-53.

6. Baskin B, Choufani S, Chen YA, Shuman C, Parkinson N, Lemyre E, et al. High frequency of copy number variations (CNVs) in the chromosome $11 \mathrm{p} 15$ region in patients with Beckwith-Wiedemann syndrome. Hum Genet. 2014;133:321-30.

7. Gurrieri F, Zollino M, Oliva A, Pascali V, Orteschi D, Pietrobono $\mathrm{R}$, et al. Mild Beckwith-Wiedemann and severe long-QT syndrome due to deletion of the imprinting center 2 on chromosome 11p. Eur J Hum Genet. 2013;21:965-9.

8. Smith AC, Suzuki M, Thompson R, Choufani S, Higgins MJ, Chiu IW, et al. Maternal gametic transmission of translocations or inversions of human chromosome $11 \mathrm{p} 15.5$ results in regional DNA hypermethylation and downregulation of CDKN1C expression. Genomics. 2012;99:25-35.

9. Demars J, Rossignol S, Netchine I, Lee KS, Shmela M, Faivre L, et al. New insights into the pathogenesis of Beckwith-Wiedemann and Silver-Russell syndromes: contribution of small copy number variations to $11 \mathrm{p} 15$ imprinting defects. Hum Mutat. 2011;32:1171-82.

10. Beygo J, Joksic I, Strom TM, Ludecke HJ, Kolarova J, Siebert R, et al. A maternal deletion upstream of the imprint control region 2 in $11 \mathrm{p} 15$ causes loss of methylation and familial Beckwith-Wiedemann syndrome. Eur J Hum Genet. 2016; 24:1280-6.

11. Paganini L, Carlessi N, Fontana L, Silipigni R, Motta S, Fiori S, et al. Beckwith-Wiedemann syndrome prenatal diagnosis by methylation analysis in chorionic villi. Epigenetics 2015; 10:643-649.

12. Eggermann T, Netchine I, Temple IK, Tumer Z, Monk D, Mackay D, et al. Congenital imprinting disorders: EUCID.net - a network to decipher their aetiology and to improve the diagnostic and clinical care. Clin Epigenetics 2015;7:23.

13. Chotalia M, Smallwood SA, Ruf N, Dawson C, Lucifero D, Frontera $\mathrm{M}$, et al. Transcription is required for establishment of germline methylation marks at imprinted genes. Genes Dev. 2009;23:105-17.

14. Smith EY, Futtner CR, Chamberlain SJ, Johnstone KA, Resnick JL. Transcription is required to establish maternal imprinting at the Prader-Willi syndrome and Angelman syndrome locus. PLoS Genet. 2011;7:e1002422.

15. Lewis MW, Brant JO, Kramer JM, Moss JI, Yang TP, Hansen PJ, et al. Angelman syndrome imprinting center encodes a transcriptional promoter. Proc Natl Acad Sci USA. 2015; 112:6871-5.

16. Singh VB, Sribenja S, Wilson KE, Attwood KM, Hillman JC, Pathak S, et al. Blocked transcription through KvDMR1 results in absence of methylation and gene silencing resembling BeckwithWiedemann syndrome. Development. 2017;144:1820-30.

17. John RM, Ainscough JF, Barton SC, Surani MA. Distant ciselements regulate imprinted expression of the mousep57(Kip2) (Cdkn1c) gene: implications for the human disorder, Beckwith-Wiedemann syndrome. Hum Mol Genet. 2001;10:1601-9.

18. Kaltenbach S, Capri Y, Rossignol S, Denjoy I, Soudee S, Aboura A, et al. Beckwith-Wiedemann syndrome and long QT syndrome due to familial-balanced translocation $\mathrm{t}(11 ; 17)(\mathrm{p} 15.5 ; \mathrm{q} 21.3)$ involving the KCNQ1 gene. Clin Genet. 2013;84:78-81.

19. Alders M, Bikker H, Christiaans I. Long QT syndrome. In: Adam MP, Ardinger HH, Pagon RA et al., editors. GeneReviews . Seattle (WA); 1993. updated 2018. 